\title{
A CLASS OF LIMIT ALGEBRAS ASSOCIATED WITH DIRECTED GRAPHS
}

\author{
DAVID W. KRIBS and BARUCH SOLEL ${ }^{\sqsubset}$
}

(Received 10 February 2005; revised 15 February 2006)

Communicated by G. Willis

\begin{abstract}
Every directed graph defines a Hilbert space and a family of weighted shifts that act on the space. We identify a natural notion of periodicity for such shifts and study their $C^{*}$-algebras. We prove the algebras generated by all shifts of a fixed period are of Cuntz-Krieger and Toeplitz-Cuntz-Krieger type. The limit $C^{*}$-algebras determined by an increasing sequence of positive integers, each dividing the next, are proved to be isomorphic to Cuntz-Pimsner algebras and the linking maps are shown to arise as factor maps. We derive a characterization of simplicity and compute the $K$-groups for these algebras. We prove a classification theorem for the class of algebras generated by simple loop graphs.
\end{abstract}

2000 Mathematics subject classification: primary 46L05, 47L 40.

Keywords and phrases: directed graph. periodic weighted shift, Fock space, limit algebra, ToeplitzCuntz-Krieger algebra, Cuntz-Pimsner algebra, factor map.

\section{Introduction}

In this paper we initiate the study of a new class of $C^{*}$-algebras associated with directed graphs. There is a family of weighted shift operators associated with every directed graph and, after identifying a natural notion of periodicity for these shifts, we conduct an in-depth analysis of their associated $C^{*}$-algebras. Specifically, we explicitly identify the structure of the $C^{*}$-algebra generated by all shifts of a given period and the limit algebras obtained from increasing sequences of positive integers, each dividing the next, strictly in terms of familiar objects from modern operator algebra theory.

Our initial motivation derives from work of Bunce and Deddens $[3,4]$ from over thirty years ago in which a class of $C^{*}$-algebras was studied via a limit algebra

(C) 2007 Australian Mathematical Society 1446-7887/07 \$A2.00+0.00 
construction that involved algebras generated by periodic weighted shift operators on a Hilbert space. The Bunce-Deddens algebras have proved to be an extremely useful, concrete class of operator algebras and have arisen in a number of diverse settings $[1,2,5,6,7,8,15,14,21,22,24,26,27]$. We were also motivated by recent work of the first author [14], where a generalization of this class was obtained for the setting of Cuntz and Toeplitz-Cuntz algebras. As we show, the class of algebras studied here contains the Bunce-Deddens algebras and the algebras from [14] as the subclass generated by single vertex directed graphs with $k$ loop edges (for $k=1$ and $k \geq 2$ respectively).

Our investigations draw on numerous aspects of contemporary operator algebra theory. We make use of fundamental results from the theory of graph $C^{*}$-algebras [25] and Cuntz-Pimsner algebras $[9,12,17,20,23]$. The theory of $C^{*}$-algebras associated with 'topological graphs', introduced by the second author and Muhly [19] and studied further in $[11,12,13,20]$, plays a central role. We utilize the theory of 'factor maps' recently invented by Katsura [12]. Each of these tools complements our predominantly spatial analysis.

The next section contains requisite preliminary material on graph $C^{*}$-algebras. We describe how weighted shifts arise from directed graphs $E$ and we identify an appropriate notion of periodicity for these shifts in Section 3. The rest of the paper contains a detailed analysis of the $C^{*}$-algebras associated with periodic shifts. In Section 4 we prove the algebras $\mathcal{A}(n)$ and $\mathcal{B}(n)$ generated by shifts of a given period are of Cuntz-Krieger and Toeplitz-Cuntz-Krieger type in such a way that the explicit connection with the underlying graph is evident. Then in Section 5 and Section 6, we identify the corresponding limit algebras $\mathcal{B}_{E}\left(\left\{n_{k}\right\}\right)$ as Cuntz-Pimsner algebras $\mathcal{O}(E(\infty))$, where the topological graph $E(\infty)$ is defined by the path structure of $E$ and the sequence $\left\{n_{k}\right\}$. In Section 7 we prove a classification theorem for the algebras $\mathcal{B}_{C_{j}}\left(\left\{n_{k}\right\}\right)$ generated by simple loop graphs $C_{j}$. We compute the $K$-groups for the algebras $\mathcal{B}_{E}\left(\left\{n_{k}\right\}\right)$ in Section 8. We finish in Section 9 by deriving a characterization of simplicity for $\mathcal{B}_{E}\left(\left\{n_{k}\right\}\right)$ in terms of $E(\infty)$ and discuss the connection with $E$.

\section{Directed graphs and their $C^{*}$-algebras}

Let $E=\left(E^{0}, E^{1}, r, s\right)$ be a directed graph with vertices $x \in E^{0}$, directed edges $e \in E^{1}$ and range and source maps $r, s$ giving the final and initial vertices of a given directed edge. We shall assume $E$ is finite and has no sources and no sinks, so that every vertex in $E^{0}$ is the initial vertex for some edge and the final vertex for some edge. The finiteness assumption is motivated by the $C^{*}$-algebra setting we work in, and the no sink assumption is motivated by our definition of periodicity. We focus on graphs with no sources simply to streamline the presentation (see Remark 6.5). 
Let $E^{*}$ be the set of all finite paths in $E$ and include the vertices $E^{0}$ in $E^{*}$ as trivial paths. Given a path $w$ in $E$ we write $w=y w x$ when the initial and final vertices of $w$ are $s(w)=x$ and $r(w)=y$, respectively. For $w$ in $E^{*}$ we write $|w|$ for the length of $w$ and put $|x|=0$ for every vertex $x \in E^{0}$. Given $n \geq 0$, let $E^{=n}$ be the set of paths in $E^{*}$ of length $n$, so that $E^{=n}=\left\{w \in E^{*}:|w|=n\right\}$. Similarly define $E^{\leq n}$ and $E^{<n}$.

There are two important $C^{*}$-algebras associated with every such graph: the CuntzKrieger algebra $C^{*}(E)$ (or $\mathcal{O}(E)$ ) and its Toeplitz extension $\mathcal{T}(E)$. For a recent survey of these algebras we point the reader to the notes [25]. Both $\mathcal{O}(E)$ and $\mathcal{T}(E)$ can be described either as universal objects or concretely. We start by recalling their universal properties.

Given a directed graph $E$, a family $\left\{P_{x}, S_{e}: x \in E^{0}, e \in E^{1}\right\}$ of projections (one for each vertex) and partial isometries (one for each edge) is said to be a ToeplitzCuntz-Krieger E-family (or a TCK E-family for short) if it satisfies the relations

$$
\begin{cases}(1) \quad P_{x} P_{y}=0 & \text { for all } x, y \in E^{0}, x \neq y, \\ \text { (2) } \quad S_{e}^{*} S_{f}=0 & \text { for all } e, f \in E^{1}, e \neq f, \\ \text { (3) } \quad S_{e}^{*} S_{e}=P_{s(e)} & \text { for all } e \in E^{1}, \\ \text { (4) } \quad \sum_{r(e)=x} S_{e} S_{e}^{*} \leq P_{x} & \text { for all } x \in E^{0} .\end{cases}
$$

Also, such a family is said to be a Cuntz-Krieger E-family (or a $C K E$-family) if equality holds in (4) whenever the set $r^{-1}(x)$ is non-empty.

The $C^{*}$-algebra $\mathcal{O}(E)$ is generated by a CK $E$-family $\left\{p_{x}, s_{e}\right\}$ and has the property that, whenever $\left\{P_{x}, S_{e}\right\}$ is a CK $E$-family inside a $C^{*}$-algebra $\mathcal{B}$, there is a *-homomorphism $\pi_{P, S}$ from $\mathcal{O}(E)$ into $\mathcal{B}$ carrying $p_{x}$ to $P_{x}$ and $s_{e}$ into $S_{e}$. The Toeplitz algebra $\mathcal{T}(E)$ has a similar universal property, but with TCK $E$-families replacing $C K E$-families.

It is also convenient to consider concrete constructions of these algebras. The details of the construction will be important when we define the generalized Bunce-Deddens algebras through a spatial approach.

Let $\mathcal{H}_{E}=\ell^{2}\left(E^{*}\right)$ be the Hilbert space with orthonormal basis $\left\{\xi_{w}: w \in E^{*}\right\}$ indexed by elements of $E^{*}$. Define a family of partial isometries on $\mathcal{H}_{E}$ as follows: for each $v \in E^{*}$ let

$$
L_{v} \xi_{w}= \begin{cases}\xi_{v w} & \text { if } s(v)=r(w), \\ 0 & \text { if } s(v) \neq r(w) .\end{cases}
$$

We use the convention $\xi_{v w}=0$ when $r(w) \neq s(v)$. We shall put $L_{x} \equiv P_{x}$ for the vertex projections.

Evidently the family $\left\{P_{x}, L_{e}\right\}$ form a TCK $E$-family. In fact, the $*$-homomorphism $\pi_{P, L}$ determined by the left regular representation is a $*$-isomorphism of $\mathcal{T}(E)$ onto 
the $C^{*}$-algebra generated by the operators $\left\{L_{e}\right\}[10,25]$. Thus, for our purposes, we may identify the algebra $\mathcal{T}(E)$ with this faithful representation $\pi_{P, L}(\mathcal{T}(E))$. We shall, therefore, for the sake of brevity define the Toeplitz algebra (concretely) as follows. The Toeplitz algebra of $E$ is the $C^{*}$-algebra

$$
\mathcal{T}(E) \equiv C^{*}\left(\left\{L_{w}: w \in E^{*}\right\}\right)=C^{*}\left(\left\{L_{e}: e \in E^{1}\right\}\right) .
$$

Let $R_{v}, v \in E^{*}$, be the partial isometries on $\mathcal{H}_{E}$ determined by the right regular representation of $E^{*}$, so that $R_{v} \xi_{w}=\xi_{w v}$. It is easy to see that the subspaces $R_{x} \mathcal{H}_{E}$ are invariant for $\mathcal{T}(E)$.

PROPOSITION 2.1. Let $\mathfrak{K}$ be the set of compact operators on $\mathcal{H}_{E}$. Then $\mathcal{T}(E)$ contains the subalgebra of compact operators $\mathfrak{K}_{E}=\oplus_{x \in E^{0}} R_{x} \mathfrak{K} R_{x}$.

PROOF. By assumption $E^{0}$ is finite and hence $\mathcal{T}(E)$ is unital as $I=\sum_{x \in E^{0}} P_{x}$. For all $x \in E^{0}$, the rank one projection $\xi_{x} \xi_{x}^{*}$ onto the subspace spanned by $\xi_{x}$ satisfies

$$
\xi_{x} \xi_{x}^{*}=P_{x}\left(\sum_{y \in E^{0}} \xi_{y} \xi_{y}^{*}\right)=P_{x}\left(I-\sum_{e \in E^{1}} L_{e} L_{e}^{*}\right) .
$$

Thus, each $\xi_{x} \xi_{x}^{*}$ belongs to $\mathcal{T}(E)$. For an arbitrary matrix unit $\xi_{v} \xi_{w}^{*}$ with $s(v)=x=$ $s(w)$ we have $\xi_{v} \xi_{w}^{*}=L_{v}\left(\xi_{x} \xi_{x}^{*}\right) L_{w}^{*} \in \mathcal{T}(E)$, and it follows that $\mathcal{T}(E)$ contains each $R_{x} \mathfrak{K} R_{x}$.

Given a scalar $z \in \mathbb{T}$ we may define a gauge unitary $U_{z} \in \mathcal{B}\left(\mathcal{H}_{E}\right)$ via

$$
U_{z} \xi_{w}=z^{|w|} \xi_{w} \quad \text { for } \quad w \in E^{*}
$$

Then $\beta_{z}\left(L_{e}\right)=U_{z} L_{e} U_{z}^{*}=z L_{e}$ defines an automorphism of $\mathcal{T}(E)$. Moreover, this automorphism leaves the ideal $\mathfrak{K}_{E}$ invariant and hence factors through to an automorphism on the quotient algebra $\mathcal{T}(E) / \mathfrak{K}_{E}$. It follows that there is a continuous gauge action $\beta: \mathbb{T} \rightarrow \operatorname{Aut}\left(\mathcal{T}(E) / \mathfrak{K}_{E}\right)$ and we obtain the following well-known result based on the 'gauge-invariant uniqueness theorem' for Cuntz-Krieger algebras [25].

THEOREM 2.2. The quotient algebra $\mathcal{T}(E) / \mathfrak{K}_{E}$ is isomorphic to the universal Cuntz-Krieger algebra $\mathcal{O}(E)$.

\section{Weighted shifts and periodicity}

Consider a finite directed graph $E=\left(E^{0}, E^{1}, r, s\right)$ with no sinks and no sources. 
DEFINITION 3.1. A family of operators $\left(T_{e}\right)_{e \in E^{1}}$ that act on $\mathcal{H}_{E}$ is a weighted shift if there are scalars $\Lambda=\left\{\lambda(w): w \in E^{*} \backslash E^{0}\right\}$ such that the operators $\left\{T_{e}: e \in E^{1}\right\}$ satisfy

$$
T_{e} \xi_{w}=\lambda(e w) \xi_{e w} \text { for all } e \in E^{1}, w \in E^{*}
$$

Now let $n \geq 1$ be a fixed positive integer. Observe that every $w \in E^{*}$ has a unique factorization of the form $w=w(n) v_{k} \cdots v_{1}$, where $v_{i} \in E^{=n}$ for $1 \leq i \leq k$ and $w(n) \in E^{<n}$.

DEFINITION 3.2. A weighted shift $T=\left(T_{e}\right)_{e \in E^{1}}$ with weights $\Lambda=\{\lambda(w): w \epsilon$ $\left.E^{*} \backslash E^{0}\right\}$ is period $n$ if $\lambda(e w)=\lambda(e w(n))$ whenever $e \in E^{1}$ and $s_{E}(e)=r_{E}(w)$. In other words, $T_{e} \xi_{w}=\lambda(e w) \xi_{e w}=\lambda(e w(n)) \xi_{e w}$ for all $e \in E^{1}, w \in E^{*}$.

DEFINITION 3.3. Let $\mathcal{A}(n)$ be the $C^{*}$-algebra generated by the $T_{e}, e \in E^{1}$, from all $n$-periodic weighted shifts $T=\left(T_{e}\right)_{e \in E^{\prime}}$ on $\mathcal{H}_{E}$. Let $\left\{n_{k}\right\}_{k \geq 1}$ be an increasing sequence of positive integers such that $n_{k} \mid n_{k+1}$ for $k \geq 1$. Observe that every period $n_{k}$ weighted. shift $T=\left(T_{e}\right)_{e \in E^{1}}$ is also period $n_{k+1}$. Thus,

$$
\mathcal{A}\left(n_{1}\right) \subseteq \mathcal{A}\left(n_{2}\right) \subseteq \cdots \subseteq \mathcal{A}\left(n_{k}\right) \subseteq \cdots,
$$

and we may consider the (norm-closed) limit algebra $\mathcal{A}_{E}\left(\left\{n_{k}\right\}\right):=\overline{\bigcup_{k \geq 1} \mathcal{A}\left(n_{k}\right)}$. As $\mathcal{A}(n)$ contains the $C^{*}$-algebra $\mathcal{T}_{E}$ generated by the unweighted shifts $L_{E}=\left(L_{e}\right)_{e \in E^{1}}$, by Proposition 2.1 it contains the compact operators $\mathfrak{K}_{E}$. Let $\mathcal{B}(n)$ be the quotient of $\mathcal{A}(n)$ by $\mathfrak{K}_{E}$, so there is a short exact sequence $0 \rightarrow \mathfrak{K}_{E} \rightarrow \mathcal{A}(n) \rightarrow \mathcal{B}(n) \rightarrow 0$. Thus, given a sequence $\left\{n_{k}\right\}_{k \geq 1}$, we have the sequence of injective inclusions

$$
\mathcal{B}\left(n_{1}\right) \subseteq \mathcal{B}\left(n_{2}\right) \subseteq \cdots \subseteq \mathcal{B}\left(n_{k}\right) \subseteq \cdots,
$$

and we may also consider the limit algebra $\mathcal{B}_{E}\left(\left\{n_{k}\right\}\right):=\overline{\bigcup_{k \geq 1} \mathcal{B}\left(n_{k}\right)}$. We refer to $\mathcal{B}_{E}\left(\left\{n_{k}\right\}\right)$ as a generalized Bunce-Deddens algebra.

\section{The algebras $\mathcal{A}(n)$ and $\mathcal{B}(n)$}

Fix a finite graph $E$ with no sinks and no sources and a positive integer $n \geq 1$. Define $E^{\text {per } n}=\left\{w \in E^{*}:|w|=m n\right.$ for some $\left.m \geq 0\right\}$.

Let $E(n)=\left(E(n)^{0}, E(n)^{1}, r_{E(n)}, s_{E(n)}\right)$ be the graph defined as follows. First define $E(n)^{0} \equiv E^{<n}$. In other words, the paths of length less than $n$ in $E$ now serve as the vertices of $E(n)$. We use $w$ to denote such a path in $E$ or a vertex in $E(n)$. It will be clear from the context what the role of $w$ is. Moreover, when we write $r_{E}(w)$ we refer 
to the vertex in $E$ that is the range of the path $w$. This vertex can be viewed either as a vertex of $E$ or as a vertex of $E(n)$ (since $\left.E^{0} \subseteq E(n)^{0}\right)$ depending on the context.

Now we set $E(n)^{1}=\left\{(e, w) \in E^{1} \times E^{<n}: s_{E}(e)=r_{E}(w)\right\}$, and the maps $r_{E(n)}$ and $s_{E(n)}$ are defined by setting $s_{E(n)}(e, w)=w$ and

$$
r_{E(n)}(e, w)= \begin{cases}e w & \text { if }|w|<n-1 \\ r_{E}(e) & \text { if }|w|=n-1\end{cases}
$$

We next define a TCK and a CK $E(n)$-family. For this we first let $T_{(e, w)}$, for $(e, w) \in E(n)^{1}$, be the operator on $\mathcal{H}_{E}$ defined by

$$
T_{(e, w)} \xi_{w^{\prime}}= \begin{cases}\xi_{e w^{\prime}} & \text { if } w=w^{\prime}(n), \\ 0 & \text { if } w \neq w^{\prime}(n)\end{cases}
$$

and $Q_{w}$, for $w \in E(n)^{0}$, be the projection onto the subspace of $\mathcal{H}_{E}$ spanned by the vectors $\xi_{w^{\prime}}$ with $w^{\prime}(n)=w$; so that

$$
Q_{w}=\cdot \sum_{w^{\prime}(n)=w} \xi_{w^{\prime}} \xi_{w^{\prime}}^{*}=\sum_{\nu \in E^{\text {শr }}, r(v)=s(w)} \xi_{w v} \xi_{w v^{*}}^{*}
$$

Observe that $T_{(e, w)}^{*} T_{(e, w)}=Q_{w}$. It is also straightforward to check that

$$
T_{(e, w)}^{*} \xi_{w^{\prime \prime}}= \begin{cases}\xi_{v} & \text { if } w^{\prime \prime}=e v, v(n)=w, \\ 0 & \text { otherwise. }\end{cases}
$$

Thus, $T_{(e, w)} T_{(e, w)}^{*}$ is the projection onto the subspace spanned by all $\xi_{w^{\prime \prime}}$ with $w^{\prime \prime}=e v$ for $v$ satisfying $v(n)=w$. It follows that, for $w_{0} \in E(n)^{0}$,

$$
\sum_{r(e, w)=w_{0}} T_{(e, w)} T_{(e, w)}^{*}= \begin{cases}Q_{w_{0}} & \text { if } n>\left|w_{0}\right|>0, \\ Q_{w_{0}}-\xi_{w_{0}} \xi_{w_{0}}^{*} & \text { if }\left|w_{0}\right|=0 .\end{cases}
$$

The index set in this sum is a singleton whenever $n>\left|w_{0}\right|>0$.

It follows that $\left\{Q_{w}, T_{(e, w)}\right\}$ is a TCK $E(n)$-family and, thus, there is a $*$-homomorphism $\rho$ from $\mathcal{T}(E(n))$ into $\mathcal{B}\left(\mathcal{H}_{E}\right)$ carrying the generators of the Toeplitz algebra to this family. Observe that every operator $T_{(e, w)}$ as above is the periodic weighted shift associated to the weights $\Lambda_{(e, w)}=\left\{\lambda\left(w^{\prime}\right): w^{\prime} \in E^{*} \backslash E^{0}\right\}$ where

$$
\lambda\left(w^{\prime}\right)= \begin{cases}1 & \text { if } w^{\prime}=e v, v(n)=w, \\ 0 & \text { otherwise }\end{cases}
$$

The operators $T_{e}$ associated with every $n$-periodic weighted shift $T=\left(T_{e}\right)_{e \in E^{\prime}}$ can be written as a finite sum

$$
T_{e}=\sum_{w \in E^{<n}, s_{E}(e)=r \varepsilon(w)} \dot{\lambda}(e w) T_{(e, w)} .
$$


It follows that the *-homomorphism $\rho$ described above maps $\mathcal{T}(E(n))$ onto $\mathcal{A}(n)$.

Setting $S_{(e, w)}=q\left(T_{(e, w)}\right)$ and $P_{w}=q\left(Q_{w}\right)$, where $q$ is the quotient map from $\mathcal{B}\left(\mathcal{H}_{E}\right)$ onto the Calkin algebra, we get a CK $E(n)$-family. Such a family defines a *-homomorphism $\pi$ from $\mathcal{O}(E(n))$ into the Calkin algebra. Since the $T_{(e, w)}$ generate $\mathcal{A}(n)$, the image of $\pi$ is $\mathcal{B}(n)$. Of course, in principle the $P_{w}$ could be zero.

LEMMA 4.1. For all $w \in E(n)^{0}, Q_{w}$ is an infinite rank projection, and hence $P_{w} \neq 0$.

PROOF. Fix $w \in E(n)^{0}$. Since $E$ has no sources, we can find paths $v_{k} \in E^{=n k}$ for $k \geq 1$ such that $s(w)=r\left(v_{k}\right)$. Then $\left(w v_{k}\right)(n)=w$ for all $k \geq 1$. Thus, $\xi_{w v_{k}}$ belongs to the range of $Q_{w}$ for all $k \geq 1$.

We may now prove the following.

THEOREM 4.2. $\pi$ is a $*$-isomorphism of $\mathcal{O}(E(n))$ onto $\mathcal{B}(n)$.

Proof. For $z \in \mathbb{T}$, let $U_{z}$ be the unitary operator on $\mathcal{H}_{E}$ defined as in (2.2). Setting $\gamma_{z}(R)=U_{z} R U_{z}^{*}$, we get a one-parameter semigroup of (inner) automorphisms of $\mathcal{B}\left(\mathcal{H}_{E}\right)$. For $(e, w) \in E(n)^{1}, w^{\prime} \in E(n)^{0}$ and $z \in \mathbb{T}$,

$$
\gamma_{z}\left(T_{(e, w)}\right) \xi_{w^{\prime}}=U_{z} T_{(e, w)} U_{z}^{*} \xi_{w^{\prime}}=z T_{(e, w)} \xi_{w^{\prime}} .
$$

Hence $\gamma_{z}\left(T_{(e, w)}\right)=z T_{(e, w)}$ and it follows that each $\gamma_{z}$ defines an automorphism of $\mathcal{A}(n)$. Moreover, as discussed above, $\gamma_{z}$ leaves $\mathfrak{K}_{E}$ invariant and so $\left\{\gamma_{z}\right\}$ induces a one parameter semigroup of automorphisms on the quotient $\mathcal{B}(n)$, which we shall also denote by $\left\{\gamma_{z}\right\}$.

Thus we have $\gamma_{z}\left(S_{(e, w)}\right)=z S_{(e, w)}$ for all $(e, w) \in E(n)^{1}$ and $\gamma_{z}\left(P_{w}\right)=P_{w}$ for all $w \in E(n)^{0}$. Since $P_{w} \neq 0$ for all $w \in E(n)^{0}$, we can now apply the gauge-invariant uniqueness theorem [25] to conclude that $\pi$ is an isomorphism.

The corresponding result with $\mathcal{A}(n)$ in place of $\mathcal{B}(n)$ does not hold (see [16] for an detailed exposition of this point). Nevertheless, there is a result for $\mathcal{A}(n)$ that is analogous to Theorem 4.2. The inspiration for the analysis sketched below comes from [12, Section 3] and [20, Section 7]. In the terminology of [20], the algebra $\mathcal{A}(n)$ is a relative quiver algebra (see also [9, Example 1.5]), related to the relative CuntzPimsner algebras introduced in [17]. Since our main focus in this paper is on the algebras $\mathcal{B}(n)$, and their direct limits, we shall only sketch the construction and the results and leave some details to the reader. The idea is to replace the graph $E(n)$ by another graph, written $E[n]$. Using the notation of [12], $E[n]$ is $E(n)_{E^{0}}$. To define it, we first let $c\left(E^{0}\right)$ be a copy of $E^{0}$ (whose elements will be written $c(v), v \in E^{0}$ ). Then $E[n]^{0}=E(n)^{0} \sqcup c\left(E^{0}\right)$ and

$$
E[n]^{1}=E(n)^{1} \sqcup\left\{(e, c(v)): e \in E^{1}, v \in E^{0}, s_{E}(e)=v\right\} .
$$


The maps $s_{E[n]}$ and $r_{E[n]}$ coincide with $s_{E(n)}$ and $r_{E(n)}$, respectively, on $E(n)^{1}$ and

$$
\begin{aligned}
& s_{E[n]}(e, c(v))=c(v) \in c\left(E^{0}\right), \\
& r_{E[n]}(e, c(v))= \begin{cases}e \in E(n)^{0} & \text { if } n>1, \\
r_{E}(e) & \text { if } n=1 .\end{cases}
\end{aligned}
$$

The TCK $E(n)$-family $\left\{Q_{w}, T_{(e, w)}\right\}$ defined above gives rise to a CK $E[n]$-family $\left\{G_{u}, R_{z}: u \in E[n]^{0}, z \in E[n]^{1}\right\}$ defined by

$$
G_{u}= \begin{cases}Q_{u}-\xi_{u} \xi_{u}^{*} & \text { if } u \in E^{0} \\ \xi_{v} \xi_{v}^{*} & \text { if } u=c(v) \in c\left(E^{0}\right) \\ Q_{u} & \text { if } u \in E(n)^{0} \backslash E^{0}\end{cases}
$$

and

$$
R_{z}= \begin{cases}T_{(e, v)}\left(Q_{v}-\xi_{v} \xi_{v}^{*}\right) & \text { if } z=(e, v) \in E(n)^{1}, v \in E^{0} \\ T_{(e, v)} \xi_{v} \xi_{v}^{*} & \text { if } z=(e, c(v)), e \in E^{1}, v \in E^{0} \\ T_{(e, w)} & \text { if } z=(e, w),(e, w) \in E(n)^{1} \backslash E^{1}\end{cases}
$$

Each of the projections $G_{u}, u \in E[n]^{0}$, is non-zero and the unitaries $U_{z}, z \in \mathbb{T}$, from (2.2) define a semigroup of gauge automorphisms on the $C^{*}$-algebra generated by $\left\{G_{u}, R_{z}\right\}$. Thus we may proceed as in Theorem 4.2 to show the following.

THEOREM 4.3. The algebra $\mathcal{A}(n)$ is $*$-isomorphic to $\mathcal{O}(E[n])$.

\section{Factor maps}

Let us examine in more detail the embedding maps that determine the limit algebras $\mathcal{B}_{E}\left(\left\{n_{k}\right\}\right)$. Fix $n, k \in \mathbb{N}$ and write $\pi_{n}: \mathcal{O}(E(n)) \rightarrow \mathcal{B}(n)$ and $\pi_{n k}: \mathcal{O}(E(n k)) \rightarrow$ $\mathcal{B}(n k)$ for the $*$-isomorphisms of Theorem 4.2 .

Recall that the algebra $\mathcal{A}(n)$ is contained in $\mathcal{A}(n k)$. We write $i_{n k, n}$ (or, simply, $i$ ) for this inclusion map and $\bar{i}_{n k, n}$ for the embedding $\bar{i}_{n k, n}: \mathcal{B}(n) \rightarrow \mathcal{B}(n k)$ induced by $i_{n k, n}$. Letting $j_{n k, n}=\pi_{n k}^{-1} \circ \bar{i}_{n k, n} \circ \pi_{n}$ we get an injective $*$-homomorphism

$$
j_{n k, n}: \mathcal{O}(E(n)) \rightarrow \mathcal{O}(E(n k)) .
$$

For $(e, w) \in E(n)^{1}, T_{(e, w)}$ is an operator from a shift of period $n$ and

$$
i_{n k, n}\left(T_{(e, w)}\right)=\sum_{\left(e, w^{\prime}\right) \in E(n k)^{1}, w^{\prime}(n)=w} T_{\left(e, w^{\prime}\right)} .
$$


Thus

$$
j_{n k, n}\left(S_{(e, w)}\right)=\sum_{\left(e, w^{\prime}\right) \in E(n k)^{1}, w^{\prime}(n)=w} S_{\left(e, w^{\prime}\right)},
$$

where here $S_{(e, w)}$ and $S_{\left(e, w^{\prime}\right)}$ are generators of $\mathcal{O}(E(n))$ and $\mathcal{O}(E(n k))$, respectively.

We now show that the map $j_{(n k, n)}$ is induced from a 'regular factor map' $m$ : $E(n k) \rightarrow E(n)$. Factor maps were introduced and studied recently by Katsura [12] in the context of topological graphs whose vertex and edge spaces are locally compact topological spaces. Here we need these concepts only for finite graphs and, thus, the definitions can be simplified.

DEFINITION 5.1. Let $F=\left(F^{0}, F^{1}, s_{F}, r_{F}\right)$ and $E=\left(E^{0}, E^{1}, s_{E}, r_{E}\right)$ be finite graphs. A factor map from $F$ to $E$ is a pair $m=\left(m^{0}, m^{1}\right)$ consisting of maps $m^{0}: F^{0} \rightarrow E^{0}$ and $m^{1}: F^{1} \rightarrow E^{1}$ such that

(i) For every $e \in F^{1}, r_{E}\left(m^{1}(e)\right)=m^{0}\left(r_{F}(e)\right)$ and $s_{E}\left(m^{1}(e)\right)=m^{0}\left(s_{F}(e)\right)$.

(ii) If $e^{\prime} \in E^{1}$ and $v \in F^{0}$ satisfy $s_{E}\left(e^{\prime}\right)=m^{0}(v)$, then there exists a unique element $e \in F^{1}$ such that $m^{1}(e)=e^{\prime}$ and $s_{F}(e)=v$.

Such a map is said to be regular if also

(iii) $\left(r_{F}\right)^{-1}(v)$ is non-empty whenever $v \in F^{0}$ and $\left(r_{E}\right)^{-1}\left(m^{0}(v)\right)$ is non-empty.

We now define $m=\left(m^{0}, m^{1}\right): E(n k) \rightarrow E(n)$ by

$$
\begin{aligned}
m^{0}(w) & =w(n) & w & \in E(n k)^{0} \quad \text { and } \\
m^{1}(e, w) & =\left(e, m^{0}(w)\right) & (e, w) & \in E(n k)^{1} .
\end{aligned}
$$

LEMMA 5.2. The pair $m=\left(m^{0}, m^{1}\right)$ defined above is a regular factor map from $E(n k)$ to $E(n)$.

ProOF. First, for $(e, w) \in E(n k)^{1}, w(n)$ is indeed in $E(n)^{0}$ (as $\left.|w(n)|<n\right)$ and $(e, w(n))$ is in $E(n)^{1}\left(\right.$ as $\left.s_{E}(e)=r_{E}(w)=r_{E}(w(n))\right)$. Fix $(e, w) \in E(n k)$. Then

$$
s_{E(n)}\left(m^{1}(e, w)\right)=s_{E(n)}(e, w(n))=w(n)=m^{0}(w)=s_{E(n k)}(e, w) .
$$

To prove a similar statement for $r$ in place of $s$ we distinguish two cases: when $|w|<n k-1$ (and so $|w(n)|<n-1$ ) and when $|w|=n k-1$ (and $|w(n)|=n-1$ ). In the first case

$$
r_{E(n)}\left(m^{1}(e, w)\right)=r_{E(n)}(e, w(n))=(e w)(n)=m^{0}(e w)=m^{0}\left(r_{E(n k)}(e, w)\right)
$$

and in the latter

$$
r_{E(n)}\left(m^{1}(e, w)\right)=r_{E(n)}(e, w(n))=r_{E}(e)=m^{0}\left(r_{E}(e)\right)=m^{0}\left(r_{E(n k)}(e, w)\right) .
$$


This establishes part (i) of the definition.

For (ii), suppose $\left(e, w^{\prime}\right) \in E(n)^{1}$ and $w_{1} \in E(n k)^{0}$ satisfy $w^{\prime}=s_{E(n)}\left(e, w^{\prime}\right)=$ $m^{0}\left(w_{1}\right)=w_{1}(n)$. Then $\left(e, w_{1}\right)$ lies in $E(n k)^{1}$, as $r_{E}\left(w_{1}\right)=r_{E}\left(w_{1}(n)\right)=r_{E}\left(w^{\prime}\right)=$ $s_{E}(e)$, and satisfies $m^{1}\left(e, w_{1}\right)=\left(e, w_{1}(n)\right)=\left(e, w^{\prime}\right)$ and $s_{E(n k)}\left(e, w_{1}\right)=w_{1}$, proving part (ii).

The claim that the map is regular follows from the fact that $E(n k)$ has no sources, since $E$ has none.

The following result is [12, Proposition 2.9] applied to finite graphs.

PROPOSITION 5.3. Let $E$ and $F$ be two finite graphs and $m$ be a regular factor map from $F$ to $E$. Then there is a unique *homomorphism $\mu_{m}: \mathcal{O}(E) \rightarrow \mathcal{O}(F)$ such that, for every $v \in E^{0}$ and $e \in E^{1}$,

(i) $\mu_{m}\left(P_{v}\right)=\sum_{u \in\left(m^{0}\right)^{-1}(v)} P_{u} ;$ and

(ii) $\mu_{m}\left(S_{e}\right)=\sum_{f \in\left(m^{1}\right)^{-1}(e)} S_{f}$.

Also, $\mu_{m}$ is injective if and only if $m^{0}$ is surjective.

Returning to $E(n k)$ and $E(n)$, together with (5.2) this result immediately implies the following.

COROLLARY 5.4. The regular factor map $m$ of Lemma 5.2 satisfies $j_{n k, n}=\mu_{m}$.

REMARK 5.5. Replacing the graphs $E(n k)$ and $E(n)$ by $E[n k]$ and $E[n]$ respectively (as in the discussion leading to Theorem 4.3), one can define a factor map $q=\left(q^{0}, q^{1}\right)$ from $E[n k]$ to $E[n]$ where $q^{i}$ agrees with $m^{i}$ on $E(n k)^{i}, i=1,2$, $q^{0}(c(v))=c(v)$ and $q^{1}(e, c(v))=(e, c(v))$. As in Corollary 5.4, the map $\mu_{q}$ induced by $q$ is the embedding of $\mathcal{O}(E[n])$ into $\mathcal{O}(E[n k])$ induced by the embedding of $\mathcal{A}(n)$ into $\mathcal{A}(n k)$.

\section{6. $\mathcal{B}_{E}\left(\left\{n_{k}\right\}\right)$ as a Cuntz-Pimsner algebra}

Fix a finite graph $E=\left(E^{0}, E^{1}, r_{E}, s_{E}\right)$ with no sinks and no sources and an increasing sequence $\left\{n_{k}\right\}_{k \geq 1}$ of positive integers with each $n_{k}$ dividing $n_{k+1}$ (and write $m_{k}=n_{k+1} / n_{k}$ ). We also write $n_{0}=1$.

It will be important for us to note that every $w \in E^{*}$ with $|w|=m$ can be written uniquely as

$$
w=w_{1} w_{2} \cdots w_{k}
$$

where

$$
w_{i} \in X_{i} \equiv\left\{w \in E^{*}: 0 \leq|w|<n_{i},|w| \equiv 0 \quad\left(\bmod n_{i-1}\right)\right\}
$$


Supposing that $\left|w_{i}\right|=k_{i} n_{i-1}$, and so $k_{i}<m_{i-1}$, we have

$$
\left|w_{i}\right|=k_{i} n_{i-1} \leq n_{i}-n_{i-1}
$$

and $m=\sum_{i=1}^{k} k_{i} n_{i-1}$. Expression (6.3) holds for all $w \in X_{i}$.

Now write $X$ for the (compact) product space $X=X_{1} \times X_{2} \times \cdots$ and $Y$ for the (closed) subset $Y=\left\{\omega=\left(w_{1}, w_{2}, \ldots\right) \in X: s_{E}\left(w_{k}\right)=r_{E}\left(w_{k+1}\right), k=1,2, \ldots\right\}$. Also, let $\tau: E^{*} \rightarrow Y$ be the map defined by

$$
\tau(w)=\left(w_{1}, w_{2}, \ldots, w_{k}, s_{E}\left(w_{k}\right), s_{E}\left(w_{k}\right), \ldots\right) \in Y,
$$

where $w=w_{1} w_{2} \cdots w_{k}$ is the decomposition as in (6.1) and (6.2). Then $\tau$ is an embedding of $E^{*}$ onto a dense subset of $Y$. We refer to $Y$ as the $\left\{n_{k}\right\}$-compactification of $E^{*}$.

DEFINITION 6.1. For $e \in E^{1}$, we define odometer maps $\sigma_{e}: D_{e} \rightarrow R_{e}$ on $Y$ as follows. First, put $D_{e}=\left\{y=\left(y_{1}, y_{2}, \ldots\right) \in Y: r_{E}\left(y_{1}\right)=s_{E}(e)\right\}$ and

$$
R_{e}=\left\{\begin{array}{l|l}
y=\left(y_{1}, y_{2}, \ldots\right) \in Y & \begin{array}{l}
\text { for some } l \leq \infty, y_{i}=r_{E}(e) \text { for all } i<l \\
\text { and }(\text { if } l \neq \infty) y_{l}=e w^{\prime} \text { for some } \\
\left|w^{\prime}\right| \equiv-1 \quad\left(\bmod n_{l-1}\right) .
\end{array}
\end{array}\right\}
$$

Now, given $\omega=\left(w_{1}, w_{2}, \ldots\right)$ in $D_{e}$, and recalling (6.3), write $i(w)$ for the smallest positive integer $i$ such that $\left|w_{i}\right|<n_{i}-n_{i-1}$ (if there is one) or $i(w)=\infty$ if $\left|w_{i}\right|=n_{i}-n_{i-1}$ for every $i$.

If $i(w)<\infty$, we write $\sigma_{e}(w)=u$, where

$$
u_{i}= \begin{cases}r_{E}(e) & \text { for } i<i(w) \\ e w_{1} \cdots w_{i(w)} & \text { for } i=i(w) \\ w_{i} & \text { for } i>i(w)\end{cases}
$$

If $i(w)=\infty$, we set $\sigma_{e}(w)=\left(r_{E}(e), r_{E}(e), \ldots\right)$.

LEMMA 6.2. For every $e \in E^{1}$,

(i) $\tau(e w)=\sigma_{e}(\tau(w))$ for every $w \in E^{*}$ such that $s_{E}(e)=r_{E}(w)$.

(ii) The sets $D_{e}$ and $R_{e}$ are compact and $\sigma_{e}$ is a continuous map from $D_{e}$ onto $R_{e}$.

Proof. Let $e \in E^{\prime}$. To prove (i), fix $w \in E^{*}$ with $s_{E}(w)=r_{E}(w)$ and let $m=i(w)$. Then $w=w_{1} \cdots w_{k}$ (as in (6.1) and (6.2)) and $\left|w_{i}\right|=n_{i}-n_{i-1}$ for $i<m$ and $\left|w_{m}\right|<n_{m}-n_{m-1}$. We have $\tau(w)=\left(w_{1}, w_{2}, \ldots, w_{k}, s_{E}(w), \ldots\right)$ and

$$
\sigma_{e}(\tau(w))=\left(r_{E}(e), r_{E}(e), \ldots, e w_{1} w_{2} \cdots w_{m}, w_{m+1}, \ldots, w_{k}, s_{E}(w), \ldots\right),
$$


where $e w_{1} \cdots w_{m}$ is in the $m$ th position. Note that $\left|w_{m}\right|=k_{m} n_{m-1}<n_{m}-n_{m-1}$ (as in (6.3)), and thus

$$
\begin{aligned}
\left|e w_{1} \cdots w_{m}\right| & =1+\left(n_{1}-1\right)+\left(n_{2}-n_{1}\right)+\cdots+\left(n_{m-1}-n_{m-2}\right)+k_{m} n_{m-1} \\
& =n_{m-1}+k_{m} n_{m-1}<n_{m} .
\end{aligned}
$$

This shows that $e w=r_{E}(e) r_{E}(e) \cdots\left(e w_{1} \cdots w_{m}\right) w_{m+1} \cdots w_{k}$ is the decomposition of $e w$ as in (6.1). It follows that $\tau(e w)=\sigma_{e}(\tau(w))$, and this establishes (i).

For (ii), since the topology on $X$ is the product topology and each $X_{i}$ is a finite set, every subset of $X$ that is defined by conditions involving only finitely many coordinates is both closed and open. Thus every subset of $Y$ defined by such conditions is closed and open in the relative topology of $Y$. This shows that $D_{e}$ is closed and open in $Y$.

For every $m \in \mathbb{N} \cup\{\infty\}$ we write $D_{m}=\left\{y \in Y: r_{E}\left(y_{1}\right)=s_{E}(e)\right.$ and $\left.i(y)=m\right\}$. Then each $D_{m}$ with $m<\infty$ is closed and open in $Y$ and the set $D_{\infty}=D_{e} \backslash \bigcup_{m<\infty} D_{m}$ is a closed set in $Y$. We also write $R_{m}(m \in \mathbb{N})$ for

$$
R_{m}=\left\{\begin{array}{l|l}
y=\left(y_{1}, y_{2}, \ldots\right) \in Y & \begin{array}{l}
y_{i}=r_{E}(e) \text { for all } i<m \text { and } y_{m}=e w^{\prime} \\
\text { for some }\left|w^{\prime}\right| \equiv-1 \quad\left(\bmod n_{m-1}\right)
\end{array}
\end{array}\right\}
$$

and $R_{\infty}=\left\{\left(r_{E}(e), r_{E}(e), \ldots\right)\right\}$. Then each $R_{m}$ (with $\left.m<\infty\right)$ is open and closed in $Y$ and $R_{\infty}$ is closed. Also $R_{e}$ is the disjoint union of all the $R_{m} \mathrm{~s}$.

Fix $m<\infty$ and define the restriction $\sigma_{m}=\left.\sigma_{e}\right|_{D_{m}}$. It is easy to see that $\sigma_{m}$ is a homeomorphism from $D_{m}$ onto $R_{m}$; in fact, it is injective and involves a change in only finitely many coordinates. We also know that $\sigma_{e}$ maps $D_{\infty}$ onto the (one-point) set $R_{\infty}$. Thus $\sigma_{e}$ maps $D_{e}$ onto $R_{e}$ and its restriction to the complement of $D_{\infty}$ is continuous.

Suppose $\left\{x^{n}\right\}$ is a sequence in $R_{e}$ converging to some $y \in Y$. If $y$ is not in $R_{\epsilon}$ then, for every $m<\infty$, only finitely many elements of the sequence lie in $R_{m}$. Thus, for every $m<\infty$, we can find some $K_{m}$ such that for every $k>K_{m}, x^{k}$ is not in $R_{i}$ for $i \leq m$. Thus, for $k>K_{m}, x_{i}^{k}=r_{E}(e)$ for all $i \leq m$. It follows that the limit, $y$, is equal to $\left(r_{E}(e), r_{E}(e), \ldots\right)$ and, thus, lies in $R_{e}$. Therefore $R_{e}$ is closed in $Y$.

It is left to show that $\sigma_{e}$ is continuous. In fact, it is left to consider sequences $\left\{z^{k}\right\}$ in $D_{e}$ converging to some $z \in D_{\infty}$. However, then the sequence $\left\{\sigma_{e}\left(z^{k}\right)\right\}$ lies in $R_{e}$. Since $Y$ is compact (and the topology is metric), we can find a converging subsequence. As the argument above shows, the limit will be in $R_{e}$ and, in fact, it will be in $R_{\infty}$ (since $z \in D_{\infty}$ ). Since $R_{\infty}$ has only one point and this point is the image of $z$ under $\sigma_{e}$, the proof is complete.

We now use the notation set above to introduce the topological graph $E(\infty)$, which plays an important role in studying the algebra $\mathcal{B}_{E}\left(\left\{n_{k}\right\}\right)$. Recall that a topological graph is given by a quadruple $F=\left(F^{0}, F^{1}, s_{F}, r_{F}\right)$ where $F^{0}, F^{1}$ are locally compact spaces, $s_{F}: F^{1} \rightarrow F^{0}$ is a local homeomorphism and $r_{F}: F^{1} \rightarrow F^{0}$ is a continuous 
map. To a topological graph $F$ one associates a graph $C^{*}$-algebra, written $\mathcal{O}(F)$ ([11] and [20]). We will not go into the details of the definition of $\mathcal{O}(F)$, but just note that it generalizes $\mathcal{O}(F)$ for finite graphs and it is the Cuntz-Pimsner $C^{*}$-algebra associated with a $C^{*}$-correspondence constructed from the graph. For the graph $E(\infty)$ that we define below, the $C^{*}$-correspondence will be discussed later (in Section 8).

Now we define the topological graph $E(\infty)$ as follows. Let

$$
E(\infty)^{0}=Y, \quad E(\infty)^{1}=\left\{(e, \omega) \in E^{1} \times Y: \omega \in D_{e}\right\},
$$

and for all $(e, \omega) \in E(\infty)^{1}$ put $s_{E(\infty)}(e, \omega)=\omega$ and $r_{E(\infty)}(e, \omega)=\sigma_{e}(\omega)$. Both $E(\infty)^{0}$ and $E(\infty)^{1}$ are compact spaces, the map $s_{E(\infty)}$ is a local homeomorphism (since its restriction to each $\{e\} \times D_{e}$ is a homeomorphism onto $D_{e}$ ) and $r_{E(\infty)}$ is continuous (since each $\sigma_{e}$ is).

Recall now that, given $n, k \in \mathbb{N}$, we defined a regular factor map $m$ from $E(n k)$ to $E(n)$ (see the discussion that precedes Lemma 5.2). With the sequence $\left\{n_{k}\right\}$ as above, we have a regular factor map from $E\left(n_{k}\right)$ to $E\left(n_{k-1}\right)$ and we denote it by $m_{k-1 . k}$. We also define, for every $k \in \mathbb{N}$, a pair $m_{k}=\left(m_{k}^{0}, m_{k}^{1}\right)$ of maps where $m_{k}^{0}$ maps $E(\infty)^{0}$ onto $E\left(n_{k}\right)^{0}$ and is defined by $m_{k}^{0}\left(w_{1}, w_{2}, \ldots\right)=w_{1} \cdots w_{k} \in E^{<n_{k}}=E\left(n_{k}\right)^{0}$ and $m_{k}^{1}$. maps $E(\infty)^{1}$ onto $E\left(n_{k}\right)^{1}$ and is defined by $m_{k}^{1}(e, \omega)=\left(e, m_{k}^{0}(\omega)\right),(e, \omega) \in E(\infty)^{1}$.

These maps are continuous and satisfy

$$
m_{k-1, k}^{0} \circ m_{k}^{0}=m_{k-1}^{0} \quad \text { and } \quad m_{k-1, k}^{1} \circ m_{k}^{1}=m_{k-1}^{1}
$$

for all $k \in \mathbb{N}$. Also, it is straightforward to check that, given a sequence $\left\{w^{(k)}\right\}$ where $w^{(k)} \in E\left(n_{k}\right)^{0}$ for all $k$ and $m_{k-1, k}\left(w^{(k)}\right)=w^{(k-1)}$, there is a unique $w \in E(\infty)$ with $m_{k}(w)=w^{(k)}$ for all $k$. Similar considerations work for the edge spaces. We also have that $s_{E\left(n_{k}\right)}\left(m_{k}^{1}(e, w)\right)=m_{k}^{0}\left(s_{E(\infty)}(e, w)\right)$ for every $(e, w) \in E(\infty)^{1}$ and a similar equality holds for the range maps. In fact, we see that $E(\infty)$ is the projective limit [12] of the projective system defined by the graphs $E\left(n_{k}\right)$ and the maps $m_{k-1, k}$ (see [12, Section 4]). Also, this projective system is surjective (in other words, each $m_{k-1, k}^{0}$ is a surjective map).

We may now prove the following.

THEOREM 6.3. Let $E$ be a finite graph with no sinks and no sources and let $\left\{n_{k}\right\}$ be an increasing sequence of positive integers with each $n_{k}$ dividing $n_{k+1}$. Then the algebra $\mathcal{B}_{E}\left(\left\{n_{k}\right\}\right)$ is *-isomorphic to the Cuntz-Pimsner $C^{*}$-algebra $\mathcal{O}(E(\infty))$.

ProOF. By [12, Theorem 4.13], $\mathcal{O}(E(\infty))$ is isomorphic to the direct limit of the algebras $\mathcal{O}\left(E\left(n_{k}\right)\right)$ with respect to the maps $\mu_{m_{k-1, k}}$. Corollary 5.4 shows that the maps $\pi_{n_{k}}$ of Theorem 4.2 can be used to get an isomorphism of this direct limit and the direct limit of the algebras $\mathcal{B}\left(n_{k}\right)$ with respect to the maps $j_{n_{k}, n_{k-1}}$. This concludes the proof since the latter algebra is $\mathcal{B}_{E}\left(\left\{n_{k}\right\}\right)=\lim _{k} \mathcal{B}\left(n_{k}\right)$. 
REMARK 6.4. One can also construct a topological graph $E[\infty]$ satisfying $\mathcal{A}(n) \cong$ $\mathcal{O}(E[n])$. It is the projective limit of the graphs $E\left[n_{k}\right]$ (see Theorem 4.3) with respect to the factor maps defined as in Remark 5.5.

REMARK 6.5. Our no source assumption on $E$ was made to clarify the presentation. In general though, one can consider the graph $\tilde{E}$ defined by $E$ as follows:

$$
\begin{aligned}
& \tilde{E}^{0}=\left\{v \in E^{0}:\left|\left\{w \in E^{*}: r_{E}(w)=v\right\}\right|=\infty\right\}, \\
& \tilde{E}^{1}=E^{1} \cap s_{E}^{-1}\left(\tilde{E}^{0}\right) \cap r_{E}^{-1}\left(\tilde{E}^{0}\right), \\
& s_{\tilde{E}}=s_{E} \mid \tilde{E}^{1} \text { and } r_{\tilde{E}}=r_{E} \mid \tilde{E}^{1} .
\end{aligned}
$$

It is straightforward to check that $\tilde{E}$ has no sources and no sinks (provided $E$ has no sinks). Also, if $E$ has no sources and no sinks then $\tilde{E}=E$.

Then for an arbitrary finite graph $E$ with no sinks (possibly with sources), $E$ may be replaced by $\widetilde{E}$ in Theorem 4.2 and Lemma 5.2. Further, in Theorem 6.3, $\mathcal{O}(E(\infty))$ may be replaced by $\mathcal{O}(\widetilde{E}(\infty))$. The following result is an immediate consequence of this generalized version of the previous theorem.

COROLLARY 6.6. If $E$ and $F$ are two finite graphs with no sinks and $\tilde{E}$ is isomorphic to $\tilde{F}$ (in particular, if $F=\tilde{E}$ ), then $\mathcal{B}_{E}\left(\left\{n_{k}\right\}\right)$ and $\mathcal{B}_{F}\left(\left\{n_{k}\right\}\right)$ are isomorphic.

\section{Example}

Let us denote by $C_{j}$ a directed graph, which is a single simple loop (or 'cycle') with $j$ vertices. In this section we shall discuss the algebras $\mathcal{B}_{C_{j}}\left(\left\{n_{k}\right\}\right)$. The algebra $\mathcal{B}_{C_{1}}\left(\left\{n_{k}\right\}\right)$ is the classical Bunce-Deddens algebra associated with the sequence $\left\{n_{k}\right\}$.

Fix a positive integer $j$. Write $v_{1}, v_{2}, \ldots, v_{j}$ for the (distinct) vertices of $C_{j}$ and $e_{1}, e_{2}, \ldots, e_{j}$ for its edges where $s\left(e_{i}\right)=v_{i}, r\left(e_{i}\right)=v_{i+1}$ if $i<j$ and $r\left(e_{j}\right)=v_{1}$.

Given a positive integer $n$, write $p$ for the least common multiple of $n$ and $j$, and $l$ for their greatest common divisor (so that $l p=j n$ ). We write $n=q j+r$ for the the division of $n$ by $j$, where $q, r$ are integers and $0 \leq r<j$. Then $\operatorname{gcd}(j, r)=\operatorname{gcd}(j, n)=l$ and, considering the equivalence relation given by addition modulo $r$ on $\{1,2, \ldots, j\}$, there are $l$ equivalence classes (each containing $j / l=p / n$ elements). Let $\Omega$ be a fixed set of representatives, one for each equivalence class.

The graphs $C_{j}(n)$ are the graphs $E(n)$ (of Section 4) with $E=C_{j}$.

LEMMA 7.1. Let $j, n$ be two positive integers. Then $C_{j}(n)$ is a disjoint union of $l$ loops, each of length p. In fact,

$$
C_{j}(n)=\bigsqcup_{i \in \Omega} C_{j}(n)^{(i)}
$$


where $C_{j}(n)^{(i)}$ is the loop that contains the vertex $v_{i}$. Thus, for each $i \in \Omega$ there is an isomorphism $\phi_{i, n}=\left(\phi_{i, n}^{0}, \phi_{i, n}^{1}\right)$ from $C_{1}(p)$ to $C_{j}(n)^{(i)}$, and we can write

$$
C_{j}(n) \cong C_{1}(p) \sqcup C_{1}(p) \sqcup \cdots \sqcup C_{1}(p),
$$

where the right-hand side is a disjoint union of l simple loops.

ProOF. For every vertex $v \in E=C_{j}$, there is a single edge ending at $v$ and a single edge emanating from it. It follows that the same holds for $C_{j}(n)$. Thus $C_{j}(n)$ is a disjoint union of loops.

Now fix $i \in \Omega$. Start with the vertex $v_{i}$ in $C_{j}(n)^{0}$, recalling that $C_{j}^{0} \subseteq C_{j}(n)^{0}$. Travelling along the edges in $C_{j}(n)^{1}$ we will, after $n-1$ 'moves' reach a vertex $w$ with $|w|=n-1$ and $s_{E}(w)=v_{i}$ (there is only one such $w$ ). From there the only way to proceed is along the edge in $C_{j}(n)^{1}$ whose source is $w$. This edge is $\left(e_{i \oplus(n-1)}, w\right)=\left(e_{i \oplus(r-1)}, w\right)$, where we write $\oplus$ for addition modulo $j$. Its range is

$$
r_{C_{j}(n)}\left(e_{i \oplus(r-1)}, w\right)=r_{C_{j}}\left(e_{i \oplus(r-1)}\right)=v_{i \oplus r} .
$$

Thus, after 'moving along' $n$ edges (starting at $v_{i}$ ) we reach the vertex $v_{i \oplus r}$. Travelling along $n$ more edges we reach $v_{2 r \oplus i}$ and so on until we get back to $v_{i}$. Clearly, $\{i, r \oplus i, 2 r \oplus i, \ldots\}$ is one of the equivalence classes mentioned above. In fact, it is the equivalence class whose representative (in $\Omega$ ) is $i$ and it contains $p / n$ elements. Thus, this loop contains $(p / n) n=p$ edges (and vertices) and we denote it by $C_{j}(n)^{(i)}$. Since this argument holds for every loop, it shows that each loop contains $p$ edges, completing the proof of the first statement of the lemma.

The last statement of the lemma follows since all simple loops of length $p$ are isomorphic.

REMARK 7.2. As mentioned in the lemma, each loop $C_{j}(n)^{(i)}$ is isomorphic to the graph $C_{1}(p)$. In fact, there are $p$ different directed graph isomorphisms from $C_{1}(p)$ to $C_{j}(n)^{(i)}$. We wish to fix one and we do so as follows. If $C_{1}^{0}=\{v\}$ and $C_{1}^{1}=\{e\}$, then a vertex in $C_{1}(p)^{0}$ is of the form $v$ or eee $\cdots e$ (with no more than $(p-1) e s$ ). For each $i \in \Omega$ we fix the only isomorphism from $C_{1}(p)$ to $C_{j}(n)^{(i)}$ that sends the vertex $v$ (in $C_{1}(p)$ ) to the vertex $v_{i}$ (in the loop $C_{j}(n)^{(i)}$ ). From now on, when we write $\phi_{i, n}=\left(\phi_{i, n}^{0}, \phi_{i, n}^{1}\right)$, we refer to this isomorphism.

LEMMA 7.3. Let $j, n, k$ be positive integers such that $\operatorname{gcd}(j, n k)=\operatorname{gcd}(j, n)($ and write $l$ for this number and $p$ for $n j / l)$. Let $m=\left(m^{0}, m^{1}\right)$ be the factor map from $C_{j}(n k)$ to $C_{j}(n)$ as in Lemma 5.2. Then, for every $i \in \Omega, m$ maps $C_{j}(n k)^{(i)}$ into $C_{j}(n)^{(i)}$. Moreover, writing $\phi_{i, n k}$ and $\phi_{i, n}$ for the isomorphisms in Remark 7.2 associated with $C_{j}(n k)^{(i)}$ and $C_{j}(n)^{(i)}$ respectively, the map $\left(\phi_{i, n}\right)^{-1} \circ m \circ \phi_{i, n k}$ is the factor map from $C_{1}(p k)$ to $C_{1}(p)$ as in Lemma 5.2 . 
PROOF. The factor map $m$, as defined in the discussion that proceeds Lemma 5.2, maps $E(n k)$ to $E(n)$ and fixes the vertices in $E^{0}$ (recall that $E^{0}$ is contained in both $E(n)^{0}$ and in $\left.E(n k)^{0}\right)$. Thus, when $E=C_{j}$, it fixes the vertices $v_{1}, \ldots, v_{j}$ and, in particular, it fixes each $v_{i}$ for $i \in \Omega$. So fix such an $i$ and write the vertices of the loop $C_{j}(n k)^{(i)}$ as $\left\{u_{1}=v_{i}, u_{2}, u_{3}, \ldots, u_{p k}\right\}$ and the vertices of $C_{j}(n)^{(i)}$ as $\left\{z_{1}=v_{i}, z_{2}, z_{3}, \ldots, z_{p}\right\}$. Also write $f_{q}$ for the edge in $C_{j}(n k)^{(i)}$ emanating from $u_{q}$ and ending at $u_{q+1}$ and, similarly write $g_{q}$ for the edge of $C_{j}(n)^{(i)}$ starting at $z_{q}$ and ending at $z_{q+1}$ (with $f_{p k}$ and $g_{p}$ defined in the obvious way).

As $m^{0}\left(u_{1}\right)=z_{1}$, it follows from (i) of Definition 5.1 that $s\left(m^{1}\left(f_{1}\right)\right)=m^{0}\left(s\left(f_{1}\right)\right)=$ $m^{0}\left(u_{1}\right)=z_{1}$ and, consequently, $m^{1}\left(f_{1}\right)=g_{1}$. Using Definition 5.1 again, we get $m^{0}\left(u_{2}\right)=m^{0}\left(r\left(f_{1}\right)\right)=r\left(m^{1}\left(f_{1}\right)\right)=r\left(g_{1}\right)=z_{2}$ (here we used $r, s$ to denote the range and source maps for both graphs, but that should cause no confusion). Continuing in this way we see that $m$ maps $C_{j}(n k)^{(i)}$ onto $C_{j}(n)^{(i)}$. In fact, the image of $m$ 'travels' along the smaller loop $k$ times.

This argument shows, in fact, that there is a unique factor map from a loop of length $p k$ to a loop of length $p$, provided we require that a chosen vertex in the first loop will be mapped to a chosen one in the second. Since $C_{1}(p k)$ and $C_{1}(p)$ are such loops and the map $\left(\phi_{i, n}\right)^{-1} \circ m \circ \phi_{i, n k}$ is a factor map from $C_{1}(p k)$ to $C_{1}(p)$ that maps the vertex $v$ (in $C_{1}(p k)$ ) to $v$ (in $C_{1}(p)$ ), it is the unique factor map that does so. It follows that it equals the factor map of Lemma 5.2 (with $C_{1}$ in place of $E$ and $p$ in place of $n$ ).

COROLLARY 7.4. Let $j, n, k$ be positive integers such that $\operatorname{gcd}(j, n k)=\operatorname{gcd}(j, n)$ (and write $l$ for this number and $p$ for $n j / l$ ). Then there are $*$-isomorphisms

$$
\Phi_{n}: \mathcal{B}_{C_{j}}(n) \rightarrow \mathcal{B}_{C_{1}}(p) \oplus \mathcal{B}_{C_{1}}(p) \oplus \cdots \oplus \mathcal{B}_{C_{1}}(p)
$$

and

$$
\Phi_{n k}: \mathcal{B}_{C_{j}}(n k) \rightarrow \mathcal{B}_{C_{1}}(p k) \oplus \mathcal{B}_{C_{1}}(p k) \oplus \cdots \oplus \mathcal{B}_{C_{1}}(p k)
$$

such that, for every $i \in \Omega,\left.j_{n k, n}^{C_{j}}\right|_{C_{j}(n)^{(i)}}=\left.\left(\Phi_{n k}\right)^{-1} \circ j_{p k, p}^{C_{1}} \circ \Phi_{n}\right|_{C_{j}(n)^{(i)}, \text { where } j_{n k, n}^{C_{j}} \text { and }}$ $j_{p k, p}^{C_{1}}$ are the maps defined in (5.1) associated with the graphs $C_{j}$ and $C_{1}$ respectively. Hence $j_{n k, n}^{C_{j}}=\left(\Phi_{n k}\right)^{-1} \circ\left(\sum_{i \in \Omega} \oplus j_{p k, p}^{C_{1}}\right) \circ \Phi_{n}$.

PROOF. The isomorphisms $\Phi_{n}$ and $\Phi_{n k}$ are the ones implemented by the graphisomorphisms $\sum_{i} \phi_{i, n}$ and $\sum_{i} \phi_{i, n k}$, respectively (these maps are defined in Remark 7.2). Since, by Corollary 5.4, the maps $j_{n k, n}^{C_{j}}$ and $j_{n k, n}^{C_{1}}$ are the ones implemented by the corresponding factor maps, the result follows from Lemma 7.3.

THEOREM 7.5. For a positive integer $j$ and a sequence of positive integers $\left\{n_{k}\right\}$ as above, the $C^{*}$-algebra $\mathcal{B}_{C_{j}}\left(\left\{n_{k}\right\}\right)$ is *-isomorphic to the direct sum of l copies of the classical Bunce-Deddens algebra $\mathcal{B}_{C_{1}}\left(\left\{p_{k}\right\}\right)$ where $l=\max _{k} \operatorname{gcd}\left(j, n_{k}\right)$ and 
$p_{k}=j n_{k} /$ l. It follows that $\mathcal{B}_{C_{j}}\left(\left\{n_{k}\right\}\right) \cong \mathcal{B}_{C_{j^{\prime}}}\left(\left\{n_{k}^{\prime}\right\}\right)$ if and only if $\max _{k} \operatorname{gcd}\left(j, n_{k}\right)=$ $\max _{k} \operatorname{gcd}\left(j^{\prime}, n_{k}^{\prime}\right)(=l)$ and the supernatural numbers associated with $\left\{j n_{k} / l\right\}$ and with $\left\{j^{\prime} n_{k}^{\prime} / l\right\}$ coincide.

PROOF. The sequence $\left\{\operatorname{gcd}\left(j, n_{k}\right)\right\}_{k=1}^{\infty}$ is a non decreasing sequence of positive integers that are smaller or equal to $j$. Thus, for some $k_{0}, \operatorname{gcd}\left(j, n_{k}\right)=l$ whenever $k \geq k_{0}$. Since we are interested in the limit algebra, we can, and shall, assume that $\operatorname{gcd}\left(j, n_{k}\right)=l$ for all $k$.

Thus, we can use Corollary 7.4 and the fact that

$$
\mathcal{B}_{C_{j}}\left(\left\{n_{k}\right\}\right)=\lim _{k}\left(\mathcal{B}_{C_{j}}\left(n_{k}\right), j_{n_{k+1}, n_{k}}^{C_{j}}\right) \quad \text { and } \quad \mathcal{B}_{C_{1}}\left(\left\{p_{k}\right\}\right)=\lim _{k}\left(\mathcal{B}_{C_{1}}\left(p_{k}\right), j_{p_{k+1}, p_{k}}^{C_{1}}\right)
$$

to conclude that the family of $*$-isomorphisms $\left\{\Phi_{n_{k}}\right\}$ (defined in Corollary 7.4) defines a *-isomorphism from $\mathcal{B}_{C_{j}}\left(\left\{n_{k}\right\}\right)$ onto the direct sum of $l$ copies of $\mathcal{B}_{C_{1}}\left(\left\{p_{k}\right\}\right)$, completing the proof of the first statement of the theorem.

Now assume that $\mathcal{B}_{C_{j}}\left(\left\{n_{k}\right\}\right) \cong \mathcal{B}_{C_{j^{\prime}}}\left(\left\{n_{k}^{\prime}\right\}\right)$. The $C^{*}$-algebra $\mathcal{B}_{C_{1}}\left(\left\{p_{k}\right\}\right)$ is the classical Bunce-Deddens algebra associated with the sequence $\left\{p_{k}\right\}$. It is known to be simple ([6, Theorem V.3.3]) and thus, the center of $\mathcal{B}_{C_{j}}\left(\left\{n_{k}\right\}\right)$ is of dimension. $l=\max _{k} \operatorname{gcd}\left(j, n_{k}\right)$ and is generated by an orthogonal family of $l$ projections whose sum is $I$. It then follows that $\max _{k} \operatorname{gcd}\left(j, n_{k}\right)=\max _{k} \operatorname{gcd}\left(j^{\prime}, n_{k}^{\prime}\right)$ (since the centers of the two algebras are isomorphic). Also, if $q$ is one of these central projections in $\mathcal{B}_{C_{j}}\left(\left\{n_{k}\right\}\right)$ and it is mapped by the isomorphism to the central projection $q^{\prime}$ in the other algebra, then the isomorphism maps $q \mathcal{B}_{C_{j}}\left(\left\{n_{k}\right\}\right) q$ (which is isomorphic to $\mathcal{B}_{C_{1}}\left(\left\{j n_{k} / l\right\}\right)$ ) onto the algebra $q^{\prime} \mathcal{B}_{C_{j}^{\prime}}\left(\left\{n_{k}^{\prime}\right\}\right) q^{\prime}$ (which is isomorphic to $\mathcal{B}_{C_{1}}\left(\left\{j^{\prime} n_{k}^{\prime} / l\right\}\right)$ ). It follows from [6, Theorem V.3.5] that the two supernatural numbers coincide. Theorem V.3.5 of [6], together with the first statement of the theorem, proves the other direction.

COROLLARY 7.6. The algebra $\mathcal{B}_{C_{j}}\left(\left\{n_{k}\right\}\right)$ is simple if and only if for every $k \geq 1$, we have $\operatorname{gcd}\left(j, n_{k}\right)=1$.

REMARK 7.7. We expect that the classification result of [14], which generalizes the Bunce-Deddens supernatural number classification to the Cuntz case, could be used to extend Theorem 7.5 to a broader class of graphs. More generally, we wonder for what graphs $E$ could a classification theorem along the lines of Theorem 7.5 be proved.

\section{8. $K$-theory}

In this section we derive the $K$-groups of the algebra $\mathcal{B}_{E}\left(\left\{n_{k}\right\}\right)$, where again $E$ is a finite graph with no sinks and no sources and $\left\{n_{k}\right\}$ is a sequence as above. We start with the $K$-theory of $C(Y)$. 
LEMMA 8.1. Let $Y$ be the $\left\{n_{k}\right\}$-compactification of $E^{*}$. Then

$$
K_{0}(C(Y)) \cong C(Y, \mathbb{Z}) \text { and } K_{1}(C(Y))=\{0\} .
$$

PROOF. For every $k \in \mathbb{N}$ write $\mathcal{C}_{k}$ for the subalgebra of all functions $f$ in $C(Y)$ with the property that $f(y)=f(z)$ whenever $y_{i}=z_{i}$ for all $i \leq k$. There is a *-isomorphism $\rho_{k}: C\left(E\left(n_{k}\right)^{0}\right) \rightarrow \mathcal{C}_{k}$ given by $\rho_{k}(g)(y)=g\left(y_{1} y_{2} \cdots y_{k}\right)$. If $\iota_{k+1, k}$ is the inclusion map of $\mathcal{C}_{k}$ into $\mathcal{C}_{k+1}$, then the map $\rho_{k+1}^{-1} \circ \iota_{k} \circ \rho_{k}$ is equal to the map $\mu_{n_{k+1}, n_{k}}^{0}$ defined above. Note that $\cup_{k} \mathcal{C}_{k}$ is a dense subalgebra of $C(Y)$ (by the Stone-Weierstrass Theorem). Thus $C(Y)=\lim _{k}\left(C\left(E\left(n_{k}\right)^{0}\right),\left(\mu_{n_{k+1}, n_{k}}\right)^{0}\right)$.

Fix $f \in C(Y)$ with values in $\mathbb{Z}$. For $0<\epsilon<1 / 2$ we can find $k$ and $g \in \mathcal{C}_{k}$ with $\|f-g\|<\epsilon$. Let $\psi: \bigcup_{n \in \mathbb{Z}}(n-\epsilon, n+\epsilon) \rightarrow \mathbb{Z}$ be defined by $\left.\psi\right|_{(n-\epsilon, n+\epsilon)} \equiv n$. Then $\psi$ is continuous and so is the function $g^{\prime}:=\psi \circ g$. However, $g^{\prime} \in \mathcal{C}_{k}$ and $f=g^{\prime}$. Thus $f \in \mathcal{C}_{k}$.

This shows that $\left\{f \in C(Y, \mathbb{Z})\right.$ : for some $\left.k, f \in \mathcal{C}_{k}\right\}=C(Y, \mathbb{Z})$. Using the notation $\left(\left(\mu_{n_{k+1}, n_{k}}\right)^{0}\right)_{*}$ for the restriction of this map to $\mathbb{Z}$-valued functions in $C\left(E\left(n_{k}\right)^{0}\right)$, we get

$$
C(Y, \mathbb{Z})=\lim _{k}\left(C\left(E\left(n_{k}\right)^{0}, \mathbb{Z}\right),\left(\left(\mu_{n_{k+1}, n_{k}}\right)^{0}\right)_{*}\right) .
$$

Since $K_{0}\left(C\left(E\left(n_{k}\right)^{0}\right)\right)$ is isomorphic to $C\left(E\left(n_{k}\right)^{0}, \mathbb{Z}\right)$ (recall that $E\left(n_{k}\right)^{0}$ is a finite set) and $\left(\left(\mu_{n_{k+1}, n_{k}}\right)^{0}\right)_{*}$ is the map induced from $\left(\mu_{n_{k+1}, n_{k}}\right)^{0}$ on the $K_{0}$ groups, we find that

$$
K_{0}(C(Y)) \cong \lim _{k}\left(C\left(E\left(n_{k}\right)^{0}, \mathbb{Z}\right),\left(\left(\mu_{n_{k+1}, n_{k}}\right)^{0}\right)_{*}\right) \cong C(Y, \mathbb{Z})
$$

Since $K_{1}\left(C\left(E\left(n_{k}\right)^{0}\right)\right)=\{0\}$ for each $k$, the second statement of the lemma also follows.

Given the topological graph $E(\infty)$, one can associate with it a $C^{*}$-correspondence $Z$ over $A=C(Y)$ as follows (see also $[11,18,20]$ ). On the space $C\left(E(\infty)^{1}\right)$ one can define a (right) $C(Y)$-module structure by setting

$$
(\psi f)(e, w)=\psi(e, w) f(w), \quad \psi \in C\left(E(\infty)^{1}\right), f \in C(Y)
$$

and a $C(Y)$-valued inner product by

$$
\left\langle\psi_{1}, \psi_{2}\right\rangle(y)=\sum_{(e, y) \in E(\infty)^{1}} \overline{\psi_{1}(y)} \psi_{2}(y)
$$

This makes $C\left(E(\infty)^{1}\right)$ into a Hilbert $C^{*}$-module over $A=C(Y)$.

To make this module into a correspondence one defines, for $f \in C(Y)$, $\psi \in C\left(E(\infty)^{1}\right),(f \psi)(e, w)=f\left(\sigma_{e}(w)\right) \psi(e, w)$. This defines the correspondence associated with this graph. It will be convenient, however, to write it in a slightly 
different way. First, for $e \in E^{1}, C\left(D_{e}\right)$ is a Hilbert $C^{*}$-module over $A=C(Y)$ and can be made into a $C^{*}$-correspondence by defining the left action using $\sigma_{e}$

$$
(f \cdot g)(y)=f\left(\sigma_{e}(y)\right) g(y), \quad f \in C(Y), g \in C\left(D_{e}\right) .
$$

Now we let $Z$ be the correspondence $Z=\oplus_{e \in E^{\prime}} C\left(D_{e}\right)$. We write $\phi_{Z}$ for the left action, that is, $\phi_{Z}(f)\left(\oplus g_{e}\right)=\oplus\left(f \circ \sigma_{e}\right) g_{e}$.

Given $\psi \in C\left(E(\infty)^{\mathfrak{l}}\right)$ and $e \in E^{1}$, write $\psi_{e} \in C\left(D_{e}\right)$ for the function $\psi_{e}(w)=$ $\psi(e, w)$. Then it is straightforward to check that the map $\psi \mapsto \oplus \psi_{e}$ is an isomorphism of correspondences from $C\left(E(\infty)^{1}\right)$ onto $Z$. Thus, we can write $Z$ for the correspondence associated with the graph $E(\infty)$.

In order to state the next result, note that $Z$ is a finitely generated Hilbert $C^{*}$ module over $A$ and the triple $\left(Z, \phi_{Z}, 0\right)$ defines an element in $K K(A, A)$. As such, it defines a map on $K_{0}(A)$ (into itself), written [Z]. In fact, a general element of $K_{0}(A)$ can be written as a difference $\left[\mathcal{E}_{1}\right]-\left[\mathcal{E}_{2}\right]$ for finitely generated projective modules $\mathcal{E}_{i}$ over $A$ and the map $[Z]$, defined by $\left(Z, \phi_{Z}, 0\right)$, will map it into the element $\left[\mathcal{E}_{1} \otimes_{A} Z\right]-\left[\mathcal{E}_{2} \otimes_{A} Z\right]$.

Using Lemma 8.1, it follows that $[Z]$ induces a map on $C(Y, \mathbb{Z})$. To see how this map is defined we first need the following discussion. Given a (finite) subset $B \subseteq E\left(n_{k}\right)^{0}$ for some $k \geq 1$ and given some $j \geq k$, we form

$$
B(j)=\left\{w \in E\left(n_{j}\right)^{0}: w\left(n_{k}\right) \in B\right\}
$$

and

$$
B(\infty)=\left\{y=\left(y_{1}, y_{2}, \ldots\right) \in Y: y_{1} y_{2} \ldots y_{k} \in B\right\} .
$$

Then $B(\infty)$ is a subset of $Y$ that is both closed and open. In fact, every subset of $Y$ that is closed and open is $B(\infty)$ for some $k$ and some subset $B$ of $E\left(n_{k}\right)^{0}$.

For such $B$ write $\chi_{B(\infty)}$ for the characteristic function of $B(\infty)$. Then $\chi_{B(\infty)} \in$ $C(Y, \mathbb{Z})$. Set $J_{B}=\{g \in C(Y): g(y)=0, y \in Y \backslash B(\infty)\}$. Then $J_{B}$ is a finitely generated projective $C(Y)$-module. Thus, it defines an element $\left[J_{B}\right]$ in $K_{0}(C(Y))$. The function in $C(Y, \mathbb{Z})$ associated with this element via the isomorphism of Lemma 8.1 is $\chi_{B(\infty)}$. To see this, write $J_{B}$ as a direct limit of $J_{B} \cap \mathcal{C}_{j}$ and note that $J_{B} \cap \mathcal{C}_{j}$ defines the element in $K_{0}\left(\mathcal{C}_{j}\right) \cong C\left(E\left(n_{j}\right)^{0}, \mathbb{Z}\right)$ that is the characteristic function of $B(j)$. For $j \geq k$ the image of this function, under the embedding of $C\left(E\left(n_{j}\right)^{0}, \mathbb{Z}\right)$ into $C(Y, \mathbb{Z})$ given by the direct limit (8.1), is the characteristic function of $B(\infty)$.

For $B$ as above and $e \in E^{0}$, consider the set $\sigma_{e}^{-1}(B(\infty))$. It is also a closed and open subset of $Y$ and, thus, is equal to $C(\infty)$ for some $k$ and $C \subseteq E\left(n_{k}\right)^{0}$. We have

$$
\begin{aligned}
J_{C} & =\{g \in C(Y): g(y)=0, y \in Y \backslash C(\infty)\} \\
& =\left\{g \in C(Y): g(y)=0, y \in Y \backslash \sigma_{e}^{-1}(B(\infty))\right\} \\
& =\left\{f \circ \sigma_{e}: f \in J_{B}\right\} .
\end{aligned}
$$


We write $J_{B} \circ \sigma_{e}$ for this space (here, and below, the function $f \circ \sigma_{e}$ is assumed to vanish outside $D_{e}$ ).

For $B$ as above we now consider $J_{B} \otimes_{A} Z$. It is straightforward to see that this Hilbert $C^{*}$-module is isomorphic to $\phi_{Z}\left(J_{B}\right) Z=\oplus_{e}\left(J_{B} \circ \sigma_{e}\right)$. It follows that $[Z]\left(\left[J_{B}\right]\right)=\sum\left[J_{B} \circ \sigma_{e}\right]$ and, viewing $[Z]$ as a map of $C(Y, \mathbb{Z})$ (via the isomorphism of Lemma 8.1), we get $[Z]\left(\chi_{B(\infty)}\right)=\sum \chi_{B(\infty)} \circ \sigma_{e}$. Since every closed and open set in $Y$ is of the form $B(\infty)$, these characteristic functions span $C(Y, \mathbb{Z})$. Thus

$$
[Z](f)=\sum_{e}\left(f \circ \sigma_{e}\right)
$$

where $f \in C(Y, \mathbb{Z})$ and $\left(f \circ \sigma_{e}\right)(y)$ is understood to be 0 if $y$ is not in $D_{e}$.

Applying a result of Katsura ([11, Corollary 6.10]) we get the following (in the notation of [11], $E(\infty)_{r g}^{0}=E(\infty)^{0}$ since $r_{E(\infty)}\left(E(\infty)^{1}\right)=E(\infty)^{0}$ and $E(\infty)^{0}$ is compact).

THEOREM 8.2 ([11]). Let $Z$ be the correspondence defined above and $[Z]$ be the map it induces in $K$-theory. Let $t^{0}$ be the imbedding of $C\left(E(\infty)^{0}\right)$ into $\mathcal{O}(E(\infty))$. Then we have the following exact sequence of $K$-groups:

$$
\begin{aligned}
& K_{0}\left(C_{0}\left(E(\infty)^{0}\right)\right) \underset{\iota_{x}-|Z|}{\longrightarrow} K_{0}\left(C_{0}\left(E(\infty)^{0}\right)\right) \underset{t_{0}^{0}}{\longrightarrow} K_{0}(\mathcal{O}(E(\infty))) \\
& \uparrow 1 \\
& K_{1}(\mathcal{O}(E(\infty))) \stackrel{t_{*}^{0}}{\longleftarrow} K_{1}\left(C_{0}\left(E(\infty)^{0}\right)\right) \stackrel{\stackrel{1 .}{-\mid}^{\longleftarrow}}{\longleftarrow} K_{1}\left(C_{0}\left(E(\infty)^{0}\right)\right) .
\end{aligned}
$$

For $f \in C(Y, \mathbb{Z})$ write $\Delta(f)=f-\sum_{\epsilon \in E^{\prime}} f \circ \sigma_{\ell}$, where $\left(f \circ \sigma_{e}\right)(y)$ is understood to be 0 if $y$ is not in $D_{e}$.

The following theorem is now a direct consequence of Theorem 8.2, Lemma 8.1 and equation (8.2).

THEOREM 8.3. The $K_{0}$ and $K_{1}$ groups of $\mathcal{B}_{E}\left(\left\{n_{k}\right\}\right)$ are given by

$$
K_{0}\left(\mathcal{B}_{E}\left(\left\{n_{k}\right\}\right)\right)=C(Y, \mathbb{Z}) / \operatorname{Im}(\Delta) \text { and } K_{1}\left(\mathcal{B}_{E}\left(\left\{n_{k}\right\}\right)\right)=\operatorname{Ker}(\Delta) .
$$

\section{Simplicity}

Simplicity of $C^{*}$-algebras associated with topological graphs was characterized in [20, Theorem 10.2] and in [13, Theorem 8.12]. We apply these results to the graph $E(\infty)$. We first need the following.

LEMMA 9.1. The graph $E(\infty)$ contains no loops. 
PROOF. Suppose $f_{1} f_{2} f_{3} \cdots f_{k}$ is a loop in $F=E(\infty)$ and let $u^{i}=s_{F}\left(f_{i}\right)$ for $1 \leq i \leq k$. Recall that $u^{i} \in Y$ and $u_{m}^{i}$ is its $m$ th coordinate. We distinguish two cases.

First suppose none of the $u^{i}$ s lie in $\tau\left(E^{0}\right)$. Then there is some $N$ such that for $m>N$ and $1 \leq i, j \leq k$,

$$
u_{m}^{i}=u_{m}^{j} .
$$

Let $g(i)=\sum_{m=1}^{N}\left|u_{1}^{i} u_{2}^{i} \cdots u_{N}^{i}\right|$, for $1 \leq i \leq k$, where $|\cdot|$ is the length of an element of $E^{*}$.

Then, for $1 \leq i<k, g(i+1)=g(i)+1>g(i)$ since $u^{i+1}=\sigma_{e}\left(u^{i}\right)$ for some $e \in E^{l}$. A similar argument shows that $g(1)>g(k)$, yielding a contradiction.

In the second case suppose one of the $u^{i}$ 's lies in $\tau\left(E^{0}\right)$. Say, $u^{1}=\tau(v)$ (for some $v \in E^{0}$ ). Write $f_{i}=\left(e_{i}, \omega_{i}\right) \in E(\infty)^{1}$ (so $e_{i} \in E^{1}$ and $\omega_{i} \in Y$ ) and then $u^{1}=\sigma_{e_{k}} \circ \sigma_{e_{k-1}} \circ \cdots \circ \sigma_{e_{1}}\left(u^{1}\right)=\tau\left(e_{k} e_{k-1} \cdots e_{1}\right)$, contradicting the fact that $u^{1} \in \tau\left(E^{0}\right)$ and $\tau$ is injective.

Since, in either case, we arrive at a contradiction, $E(\infty)$ contains no loops.

Using the notation of [13], it now follows immediately that $E(\infty)$ is what Katsura calls a 'topologically free graph' and, in the notation of [20], the graph satisfies' Condition (L).

In order to discuss simplicity we need also the notion of minimality. This is defined in both [13] and [20]. For the graph $E(\infty)$ both definitions are easily seen to be equivalent to the following.

DEFINITION 9.2. A subset $B \subseteq E(\infty)^{0}=Y$ is said to be invariant if $\sigma_{e}(y) \in B$ whenever $y \in B \cap D_{e}$ and there is some $f \in E^{1}$ and $z \in D_{f} \cap B$ such that $\sigma_{f}(z)=y$. The graph $E(\infty)$ is said to be minimal if there is no proper, nonempty, closed invariant subset of $Y$.

The following is a consequence of Lemma 9.1 and [13, Theorem 8.12] or [20, Theorem 10.2].

THEOREM 9.3. The algebra $\mathcal{B}_{E}\left(\left\{n_{k}\right\}\right)$ is simple if and only if $E(\infty)$ is minimal.

We would like, of course, to have a condition on $E$ and the sequence $\left\{n_{k}\right\}$ that is necessary and sufficient for the minimality of $E(\infty)$. So far, we do not have such a condition for arbitrary graphs but we present a sufficient condition below. We shall need the following lemma.

LEMMA 9.4. Every nonempty closed (with respect to $E(\infty)$ ) invariant subset $Y_{0} \subseteq Y$ contains an element of the form $\tau(u)$ for some $u \in E^{0}$. Moreover, for such $u, Y_{0}$ contains every $\tau(w)$ for $w \in E^{*}$ with $s_{E}(w)=u$. 
PROOF. Let $Y_{0}$ be a closed invariant subset of $Y$. Fix $y \in Y_{0}$ and write it $y=\left(y_{1}, y_{2}, \ldots\right)$. We can write $y_{1}=e_{1} \cdots e_{j}$ for some $e_{1}, \ldots, e_{j} \in E^{1}$. Then $y=\sigma_{e_{1}}\left(e_{2} e_{3} \cdots e_{j}, y_{2}, \ldots\right)$ and the element $z=\left(e_{2} e_{3} \cdots e_{j}, y_{2}, \ldots\right)$ is the unique one satisfying $y=\sigma_{e}(z)$ for some $e \in E^{1}$. It follows from the invariance of $Y_{0}$ that $z \in Y_{0}$. Continuing in this way and noting that $y=\sigma_{e_{1}} \circ \cdots \circ \sigma_{e_{j}}\left(s_{E}\left(e_{j}\right)=r_{E}\left(y_{2}\right), y_{2}, \ldots\right)$, we find that

$$
y[1]:=\left(r_{E}\left(y_{2}\right), y_{2}, \ldots\right) \in Y_{0} .
$$

If $y[1] \in \tau\left(E^{0}\right)$, we are done. Otherwise, we write $y_{2}=e_{1}^{\prime} \cdots e_{l}^{\prime}$ for $e_{1}^{\prime}, \ldots e_{l}^{\prime} \in E^{\prime}$. Note that $y[1]=\sigma_{e_{1}^{\prime}} \circ \cdots \circ \sigma_{e_{1}^{\prime}}\left(r_{E}\left(y_{3}\right), r_{E}\left(y_{3}\right), y_{3}, \ldots\right)$, and we conclude from the invariance of $Y_{0}$ that

$$
y[2]:=\left(r_{E}\left(y_{3}\right), r_{E}\left(y_{3}\right), y_{3}, \ldots\right) \in Y_{0} .
$$

Continuing in this way, we get a sequence $y[k]$ in $Y_{0}$ with

$$
y[k]=\left(r_{E}\left(y_{k+1}\right), \ldots, r_{E}\left(y_{k+1}\right), y_{k+1}, y_{k+2}, \ldots\right) .
$$

Since $E$ is a finite graph, one of the vertices, say $u \in E^{0}$, will appear infinitely many times in the sequence $\left\{r_{E}\left(y_{k+1}\right)\right\}$. So, for some increasing sequence of positive integers $\left\{k_{m}\right\}, r_{E}\left(y_{k_{m}+1}\right)=u$ for every $m$. It follows that the sequence $y\left[k_{m}\right]$ converges in $Y$ to $(u, u, \ldots)=\tau(u)$. Since $Y_{0}$ is closed, $\tau(u)$ belongs to $Y_{0}$.

For the last statement of the lemma, fix $w \in E^{*}$ with $s_{E}(w)=u$ and write it $w=e_{1} e_{2} \cdots e_{k}$ (with $e_{i} \in E^{1}$ and $s_{E}\left(e_{k}\right)=u$ ). Then $\tau(w)=\sigma_{e_{1}} \circ \sigma_{e_{2}} \cdots \circ \sigma_{e_{k}}(\tau(u)$ ) and it follows from the invariance of $Y_{0}$ that $\tau(w) \in Y_{0}$.

PROPOSITION 9.5. If, for every $v, u$ in $E^{0}$ and every $k \geq 1$, there is some $w \in E^{*}$ with $s_{E}(w)=v, r_{E}(w)=u$ and $|w|$ is a multiple of $n_{k}$, then the algebra $\mathcal{B}_{E}\left(\left\{n_{k}\right\}\right)$ is simple.

PROOF. Suppose the condition in the hypothesis holds, and fix a closed invariant nonempty subset $Y_{0}$ of $Y$. We shall show that $Y_{0}=Y$. Since $\tau\left(E^{*}\right)$ is dense in $Y$, it suffices to show that $\tau\left(E^{*}\right) \subseteq Y_{0}$. From Lemma 9.4 we conclude that there is some $u \in E^{0}$ such that $\tau(w) \in Y_{0}$ whenever $w \in E^{*}$ with $s_{E}(w)=u$.

Now we fix $v \in E^{0}$ and a positive integer $k$. By assumption, it follows that there is some $w[k] \in E^{*}$ with $s_{E}(w[k])=u, r_{E}(w[k])=v$ and $|w[k]|$ is a multiple of $\boldsymbol{n}_{k}$. Then $y[k]:=\tau(w[k])$ has the form

$$
y[k]=\left(v, v, \ldots, v, w_{k+1}, w_{k+2}, \ldots w_{m}, u, u, \ldots\right),
$$

where $w[k]=v v \cdots v w_{k+1} w_{k+2} \cdots w_{m}$ is the decomposition of $w[k]$ as in (6.1). It follows that $y[k] \rightarrow(v, v, \ldots)=\tau(v)$, proving that $\tau(v) \in Y_{0}$. The argument of the last paragraph of the proof of Lemma 9.4 shows now that $Y_{0}$ contains every $\tau\left(w^{\prime}\right)$ with $s_{E}\left(w^{\prime}\right)=v$. Since $v$ is arbitrary, $\tau\left(E^{*}\right) \subseteq Y_{0}$, and this completes the proof. 
Recall that in Corollary 7.6 we showed that simplicity for the algebras $\mathcal{B}_{C_{j}}\left(\left\{n_{k}\right\}\right)$ depends only on $j$ and $\left\{n_{k}\right\}$. This dependence vanishes when $C_{j}$ is slightly adjusted.

COROLLARY 9.6. Let $E$ be a graph that consists of a simple loop with at least one loop edge attached at some vertex. Then $\mathcal{B}_{E}\left(\left\{n_{k}\right\}\right)$ is simple for every choice of sequence $\left\{n_{k}\right\}$.

\section{Acknowledgements}

We would like to thank Allan Donsig and Michael Lamoureux for organizing a workshop at the Banff International Research Station (December 2003) and Gordon Blower and Stephen Power for organizing a conference in Ambleside, UK (July 2004) that helped foster this collaboration. The first author was partially supported by an NSERC grant. The second author was supported by the Fund for the Promotion of Research at the Technion.

\section{References}

[1] R. J. Archbold, 'An averaging process for $C^{*}$-algebras related to weighted shifts', Proc. London Math. Soc. (3) 35 (1977), 541-554.

[2] B. Brenken. ' $C^{*}$-algebras associated with topological relations', J. Ramanujan Math. Soc. 19 (2004), 35-55.

[3] J. Bunce and J. Deddens, ' $C^{*}$-algebras generated by weighted shifts', Indiana Univ. Math. J. 23 (1973), 257-271.

[4] _ - 'A family of simple $C^{*}$-algebras related to weighted shift operators', J. Funct. Anal. 19 (1975), 13-24.

[5] M. Dadarlat and G. Gong, 'A classification result for approximately homogeneous $C^{*}$-algebras of real rank zero', Geom. Funct. Anal. 7 (1997), 646-711.

[6] K. Davidson, $C^{*}$-algebras by example, Fields Institute Monographs 6 (Amer. Math. Soc., Providence, RI, 1996).

[7] G. A. Elliott, G. Gong, H. Lin and C. Pasnicu, 'Abelian $C^{*}$-subalgebras of $C^{*}$-algebras of real rank zero and inductive limit $C^{*}$-algebras', Duke Math. J. 85 (1996), 511 1-554.

[8] D. Evans, 'Gauge actions on $\mathcal{O}_{A}$ ', J. Operator Theory 7 (1982), 79-100.

[9] N. Fowler, P. Muhly and I. Raeburn, 'Representations of Cuntz-Pimsner algebras', Indiana Univ. Math. J. 52 (2003), 569-605.

[10] N. Fowler and I. Raeburn, 'The Toeplitz algebra of a Hilbert bimodule', Indiana Univ. Math. J. 48 (1999), 155-181.

[11] T. Katsura, 'A class of $C^{*}$-algebras generalizing both graph algebras and homeomorphism $C^{*}$-algebras. I. Fundamental results'. Trans. Amer. Math. Soc. 356 (2004), 4287-4322.

[12] - 'A class of $C^{*}$-algebras generalizing both graph algebras and homeomorphism $C^{*}$-algebras. II. Examples', Internat. J. Math. 17 (2006), 791-833.

[13] _ 'A class of $C^{*}$-algebras generalizing both graph algebras and homeomorphism $C^{*}$-algebras. III. Ideal structures', Ergodic Theory Dyn. Sys. 26 (2006), 1805-1854. 
[14] D. W. Kribs, 'Inductive limit algebras from periodic weighted shifts on fock space', New York J. Math. 8 (2002), 145-159.

[15] _ 'On bilateral weighted shifts in noncommutative multivariable operator theory', Indiana Univ. Math. J. 52 (2003), 1595-1614.

[16] D. W. Kribs and B. Solel, 'A class of limit algebras associated with directed graphs', Preprint (math.OA/0411379).

[17] P. Muhly and B. Solel, 'Tensor algebras over $C^{*}$-correspondences: representations, dilations, and $C^{*}$-envelopes', J. Funct. Anal. 158 (1998), 389-457.

[18] - 'Tensor algebras, induced representations, and the Wold decomposition', Canad. J. Math. 51 (1999), 850-880.

[19] - 'On the Morita equivalence of tensor algebras', Proc. London Math. Soc. (3) 81 (2000), 113-168.

[20] P. Muhly and M. Tomforde, ‘Topological quivers', Internat. J. Math. 16 (2005), 693-755.

[21] M. O'uchi, ' $C^{*}$-bundles associated with generalized Bratteli diagrams', Internat. J. Math. 9(1998), 95-105.

[22] C. Pasnicu, 'Automorphisms of inductive limit $C^{*}$-algebras', Math. Scand. 74 (1994), 263-270.

[23] M. Pimsner, 'A class of $C^{*}$-algebras generalizing both Cuntz-Krieger algebras and crossed products by $\mathbb{Z}^{\prime}$, in: Free probability theory (Waterloo, ON, 1995), Fields Inst. Commun. 12 (Amer. Math. Soc., Providence, RI, 1997) pp. 189-212.

[24] S. C. Power, 'Non-self-adjoint operator algebras and inverse systems of simplicial complexes', J. Reine Angew: Math. 421 (1991), 43-61.

[25] I. Raeburn, Graph algebras, Conference Board of the Mathematical Sciences (Amer. Math. Soc., Providence, RI, 2005).

[26] M. Rordam, 'Classification of nuclear, simple $C^{*}$-algebras', in: Classification of nuclear $C^{*}$-algebras. Entropy in operator algebras, Encyclopedia Math. Sci. 126 (Springer, Berlin, 2002) pp. 1-145.

[27] B. Solel, 'Limit algebras associated with an automorphism'. Math. Scand. 95 (2004), 101-123.

Department of Mathematics and Statistics

University of Guelph

Guelph

Ontario N1G 2W1

Canada

e-mail:dkribs@uoguelph.ca
Department of Mathematics Technion - Israel Institute of Technology Haifa 32000 Israel e-mail:mabaruch@techunix.technion.ac.il 